\title{
Bauermeister Score
}

National Cancer Institute

\section{Source}

National Cancer Institute. Bauermeister Score. NCI Thesaurus. Code C154808.

A score based on the Bauermeister scale, a 5-point grading system used to rate the extent of myelofibrosis in potential myeloproliferative neoplasms, based on the amount of reticulin and collagen fibers present in the bone marrow. (Bauermeister DE.

Quantitation of bone marrow reticulin-a normal range. Am J Clin Pathol. 1971 Jul;56(1):2431.) 